\title{
Increased low field magnetoresistance in electron doped system $\mathrm{Sr}_{0.4} \mathrm{Ba}_{1.6-\mathrm{x}} \mathrm{La}_{\mathrm{x}} \mathrm{FeMoO}_{6}$
}

\author{
Vibhav Pandey, ${ }^{1}$ Vivek Verma, ${ }^{1}$ G. L. Bhalla, ${ }^{2}$ and R. K. Kotnala ${ }^{1, a)}$ \\ ${ }^{1}$ National Physical Laboratory, New Delhi 110012, India \\ ${ }^{2}$ Department of Physics and Astrophysics, University of Delhi, New Delhi 110007, India
}

(Received 27 March 2010; accepted 20 July 2010; published online 13 September 2010)

\begin{abstract}
Magnetotransport properties of electron doped polycrystalline system $\mathrm{Sr}_{0.4} \mathrm{Ba}_{1.6-\mathrm{x}} \mathrm{La}_{\mathrm{x}} \mathrm{FeMoO}_{6}$ are presented in this paper. We have observed increased low field magnetoresistance values with significant Curie temperature increase in the electron doped system $\mathrm{Sr}_{0.4} \mathrm{Ba}_{1.6-\mathrm{x}} \mathrm{La}_{\mathrm{x}} \mathrm{FeMoO}_{6}$. The low field magnetoresistance value (at $1000 \mathrm{Oe}$ ) in $20 \% \mathrm{La}^{3+}$ doped sample is observed to be $2.17 \%$ at $300 \mathrm{~K}$. At $0.64 \mathrm{~T}$ and $80 \mathrm{~K}$, the magnetoresistance change measured in this sample is $21.4 \%$. This sample also showed $50 \mathrm{~K}$ increase in Curie temperature over the pristine sample. The increased low field magnetoresistance values are associated with modified grain boundary barriers of the system. The results confirm the fact that the modification of grain boundary barriers has enough potential to possess high low field magnetoresistance values even in the systems with lower spin polarization values. The role of grain to grain connectivity is observed to be dominantly determining the low field magnetoresistance values in grain to grain tunnel type magnetoresistance. (c) 2010 American Institute of Physics. [doi:10.1063/1.3481085]
\end{abstract}

\section{INTRODUCTION}

Half metallic materials with high Curie temperature and high low field magnetoresistive values are very important for room temperature spintronics applications as well as other high temperature sensor devices. Some polycrystalline double perovskites $\mathrm{A}_{2} \mathrm{FeMoO}_{6}$ systems has been proved to be materials exhibiting high Curie temperature and high low field magnetoresistance (LFMR) values, where the magnetoresistive response originates from spin polarized carrier tunneling through insulating grain boundary barriers. ${ }^{1,2}$ High spin polarization and insulating grain boundary barriers in these systems enables them to exhibit high LFMR values while their high Curie temperature allows the application well above room temperature also. Electron doping through trivalent substitution at divalent "A" site has been proved to be one of the best way to increase the Curie temperature in these compounds. ${ }^{3-7}$ Trivalent doping adds one electron to these double perovskite systems which preferably occupy Mo $4 d \downarrow$ site. Further the calculations predicted that the down spin band (hybridized $\mathrm{Fe} 3 d$, O $2 p$, and Mo $4 d$ ) in these systems crosses the Fermi level. ${ }^{1,2}$ Therefore, the electron added has itinerant character and enhances antiferromagnetic interaction between itinerant electrons and localized electrons at $\mathrm{Mo}$ and $\mathrm{Fe}$ sites. This increased antiferromagnetic interaction increases the Curie temperature of these compounds. However, the results also predict the unusual filling of both up and down bands with electron doping ${ }^{8,9}$ which reduces spin polarization in the system. Also a decrease in Mo valence state with electron doping further reduces charge difference between $\mathrm{Fe}$ and Mo site, and leads to increase in disorder defects in the system ultimately reducing the spin polarization of the system. ${ }^{6,9,10}$ Although, the antisite disorder defect values can be controlled thermody-

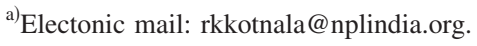

namically in electron doped systems, ${ }^{5}$ however, the loss of spin polarization cannot be prevented. In summary, the most commonly observed results with electron doping in these systems are the increase in Curie temperature, decrease in saturation magnetization, reduced spin polarization, and finally reduced magnetoresistive response of the system. ${ }^{3-10}$ The decreased spin polarization and poor LFMR are the main drawbacks of electron doping for such compounds. Due to these drawbacks, the applicability of electron doping is often questioned. ${ }^{4,11}$ In this paper, we have reported an increased low field magnetoresistive response in electron doped double perovskite system $\mathrm{Sr}_{0.4} \mathrm{Ba}_{1.6-\mathrm{x}} \mathrm{La}_{\mathrm{x}} \mathrm{FeMoO}_{6}$ and the results are correlated with the observed modification of grain boundary barriers.

\section{EXPERIMENTAL}

The polycrystalline samples of $\mathrm{Sr}_{0.4} \mathrm{Ba}_{1.6-\mathrm{x}} \mathrm{La}_{\mathrm{x}} \mathrm{FeMoO}_{6}$ were prepared by standard solid-state reaction method. Powders of high purity $\mathrm{BaCO}_{3}, \mathrm{SrCO}_{3}, \mathrm{La}_{2} \mathrm{O}_{3}, \mathrm{Fe}_{2} \mathrm{O}_{3}$, and $\mathrm{MoO}_{3}$ were mixed, ground, and calcined at $900{ }^{\circ} \mathrm{C}$ in Ar atmosphere for $10 \mathrm{~h}$. After grinding the calcined powder mixture was pressed into pellets. The pellets were then sintered at $1150{ }^{\circ} \mathrm{C}$ for $10 \mathrm{~h}$ in a gas flow of $5 \% \mathrm{H}_{2}$ and $95 \% \mathrm{Ar}$. Structural characterization were carried out using X-ray powder diffractometer at room temperature. The saturation magnetic moment value and magnetization versus temperature plots were determined by vibrating sample magnetometer. The Curie temperature of the samples was calculated by extrapolating the maximum slope to minimum of magnetization level. The electrical and magnetotransport properties were determined using four probe technique. 
TABLE I. Different properties of the sample $\mathrm{Sr}_{0.4} \mathrm{Ba}_{1.6-\mathrm{x}} \mathrm{La}_{\mathrm{x}} \mathrm{FeMoO}_{6}$.

\begin{tabular}{|c|c|c|c|c|c|c|c|c|c|c|}
\hline \multirow[b]{2}{*}{$\mathrm{X}$} & \multicolumn{2}{|c|}{$\begin{array}{c}\text { Ms } \\
\mu_{\mathrm{B}} / \text { f.u. }\end{array}$} & \multirow{2}{*}{$\begin{array}{l}\mathrm{Tc} \\
(\mathrm{K})\end{array}$} & \multirow{2}{*}{$\begin{array}{c}\mathrm{Hc}(\mathrm{Oe}) \\
300 \mathrm{~K}\end{array}$} & \multicolumn{2}{|c|}{$\mathrm{MR} \%(0.64 \mathrm{~T})$} & \multirow{2}{*}{$\begin{array}{c}\text { MR\% }(1000 \mathrm{Oe}) \\
300 \mathrm{~K}\end{array}$} & \multirow[b]{2}{*}{$\mathrm{Chi}^{2}$} & \multirow{2}{*}{$\begin{array}{l}\mathrm{a}^{\mathrm{a}} \\
(\AA)\end{array}$} & \multirow{2}{*}{$\begin{array}{r}\mathrm{ASD}^{\mathrm{b}} \\
(\%)\end{array}$} \\
\hline & $300 \mathrm{~K}$ & $80 \mathrm{~K}$ & & & $300 \mathrm{~K}$ & $80 \mathrm{~K}$ & & & & \\
\hline 0.0 & 1.48 & 3.17 & 340 & 27 & 3.72 & 8.96 & 0.88 & 1.96 & 8.0425 & 6 \\
\hline 0.1 & 1.81 & 3.12 & 354 & 25 & 4.17 & 9.27 & 0.89 & 2.01 & 8.0367 & 7 \\
\hline 0.2 & 1.93 & 3.06 & 367 & 35 & 3.64 & 14.6 & 0.95 & 1.85 & 8.0245 & 4 \\
\hline 0.3 & 2.06 & 3.05 & 378 & 39 & 5.12 & 17.23 & 1.83 & 2.12 & 8.015 & 5 \\
\hline 0.4 & 1.91 & 2.67 & 390 & 47 & 3.32 & 21.43 & 2.15 & 2.07 & 8.0043 & 7 \\
\hline
\end{tabular}

$\mathrm{a} \cong 2 d_{\langle\mathrm{Fe}-\mathrm{O}-\mathrm{Mo}\rangle}$.

${ }^{\mathrm{b}}$ Antisite disorder defect.

\section{RESULTS AND DISCUSSION}

The x-ray diffraction patterns of all the samples prepared confirms that the samples are single phase except a small amount of impurity phase $\mathrm{SrO}_{1.9}$ is present in all the samples. The structural analyses were done using Rietveld analysis also and the results are shown in Table I. Figure 1 shows the Rietveld fitting for $\mathrm{x}=0.0$ and 0.4 compounds. The substitution of smaller size $\mathrm{La}^{3+}$ in place of $\mathrm{Ba}^{2+}$ ion leads to decrease in lattice parameters. The analysis also shows that the antisite disorder defect values in the compounds are not varying much, even in highest electron doped system these are below 7\% (Table I). The calculated disorder defects values of the samples are in well agreement with saturation magnetization values of the samples.

The magnetizations versus magnetic field plots of all the samples are shown in Fig. 2. Inset in Fig. 2 shows the magnetization versus temperature plots for different samples. All the samples exhibit soft ferromagnetic nature with small coercivity and remanance values. The saturation magnetic moment value is observed decreasing with increasing doping concentration. The electron doped to system couples antiferromagnetically to Fe core spins and this increased antiferromagnetic interaction between localized and doped itinerant electron decreases the magnetic moment value of the compound. ${ }^{4,6,12}$ A significant increase in Curie temperature has been observed due to $\mathrm{La}^{3+}$ substitution. The Curie temperature increase associated with $\mathrm{La}^{3+}$ substitution is 2.5

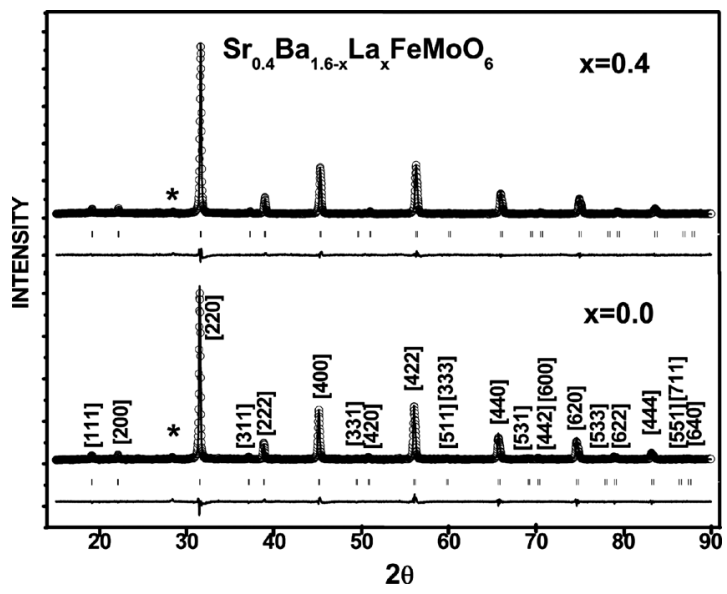

FIG. 1. Rietveld fitting for compound $\mathrm{x}=0.0$ and 0.4 . The symbol “*”, shows the impurity phase $\mathrm{SrO}_{1.9}$.
$\mathrm{K} / \%$ of La doping. The Curie temperature in these systems depends on the strength of antiferromagnetic coupling between $\mathrm{Fe}$ and Mo sublattices ${ }^{2}$ which in turn depend on $\mathrm{Fe}-$ O-Mo hybridization and carrier concentration at Fermi level. The Curie temperature increase observed in case of $\mathrm{La}^{3+}$ doping is contributed by both of these effects. First, the structural effects through increase in steric pressure which increases $\mathrm{Fe}-\mathrm{Mo}$ hybridization through decreased $\mathrm{Fe}-\mathrm{O}-\mathrm{Mo}$ distance. Second, the electron doping effects due to trivalent ion substitution at divalent " $\mathrm{A}$ " site increases the carrier electron concentration through electron filling of the bands. These effects are observed and investigated by many groups. $^{4,6,11,13}$

The MR behavior of the samples at 80 and $300 \mathrm{~K}$ are shown in Figs. 3(a) and 3(b). At low temperature, the MR values (at $0.64 \mathrm{~T}$ ) are higher in electron doped system. Surprisingly, a continuous increase in low temperature MR values is observed in whole doping range. Although the MR values at room temperature (and $0.64 \mathrm{~T}$ ) are not increasing in whole doping range (Table I, maximum MR for $\mathrm{x}=0.30$ ), however, similar as at low temperature the MR values below 1000 Oe are higher in electron doped systems [Fig. 3(b) and Table I]. The observed increase in MR values with electron doping is surprising and also inconsistent with earlier published literature. ${ }^{4,7,8}$ These reports claim a decrease in MR values with increase in electron doping. In general for electron doped double perovskite systems, the decrease in MR arises mainly due to the decrease in spin polarization result-

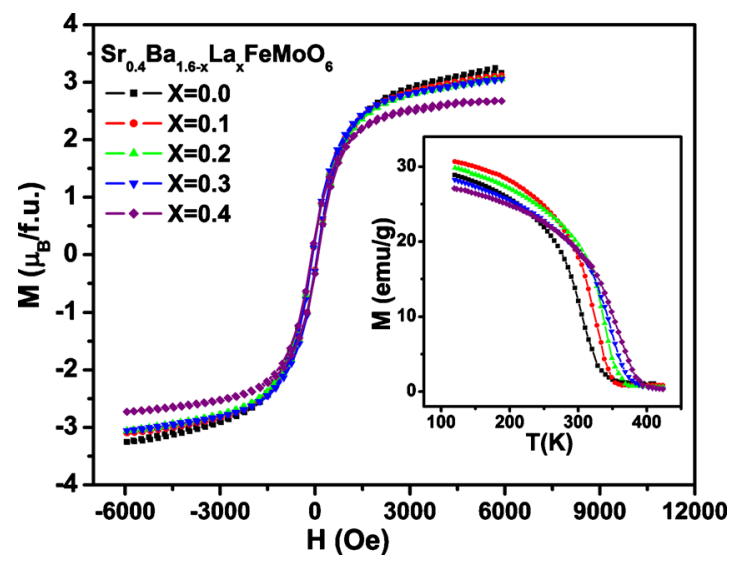

FIG. 2. (Color online) Magnetization vs applied magnetic field plots for all the $\mathrm{Sr}_{0.4} \mathrm{Ba}_{1.6-\mathrm{x}} \mathrm{La}_{\mathrm{x}} \mathrm{FeMoO}_{6}$ samples. Inset shows the magnetization vs temperature plots for the samples. 

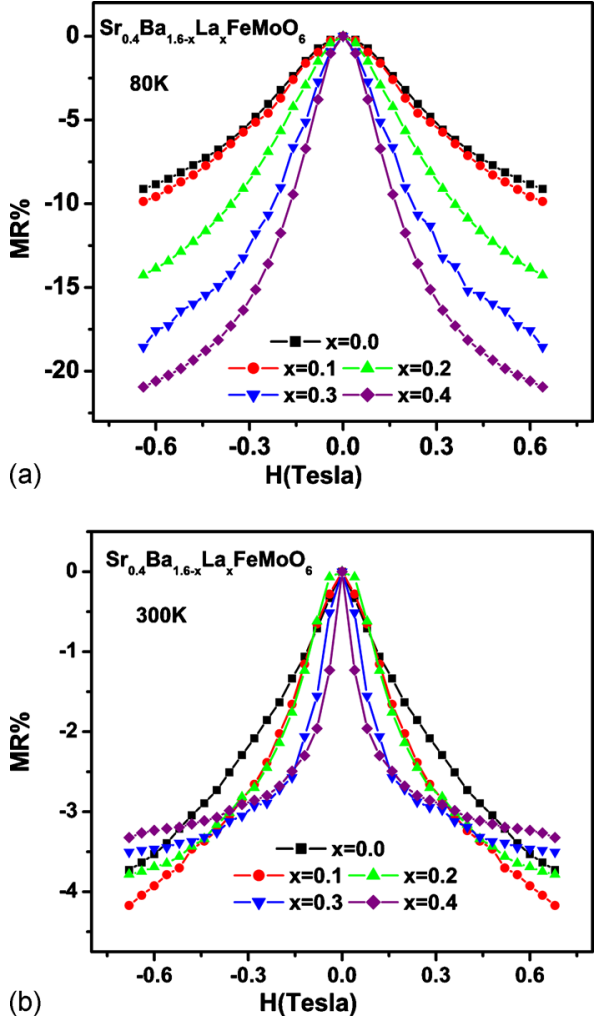

FIG. 3. (Color online) (a) MR values of the samples $\mathrm{Sr}_{0.4} \mathrm{Ba}_{1.6-\mathrm{x}} \mathrm{La}_{\mathrm{x}} \mathrm{FeMoO}_{6}$ at $80 \mathrm{~K}$. (b) MR values of the samples $\mathrm{Sr}_{0.4} \mathrm{Ba}_{1.6-\mathrm{x}} \mathrm{La}_{\mathrm{x}} \mathrm{FeMoO}_{6}$ at $300 \mathrm{~K}$.

ing from unusual filling of bands and increase in disorder defects. ${ }^{4,8}$ In view of these reports the increase in MR with electron doped systems is unusual. The more important factor in present study is the increase in low field component of MR (MR below 1000 Oe) at room temperature. An increase in MR with electron doping is also reported by Rao et al. ${ }^{3}$ and a random disorder of spin orientation was expected to be the origin of MR increase.

The basic explanation about large LFMR in these polycrystalline double perovskite systems is the tunneling conductance of spin polarized charge carriers between grains. The tunnel process takes place across grains separated by a barrier (grain boundaries of few nanometer thicknesses). The tunneling probability contains a magnetic term related to relative orientations of the magnetizations at each side of the barriers. The tunneling probability of spin polarized charge carriers is high for parallel oriented magnetizations of grains. An applied magnetic field aligns the magnetizations at two sides of the barriers which ultimately results in increased tunneling of the carriers giving a drop in resistance value (tunneling MR). In such type of tunneling conduction the important factors affecting the tunneling MR are the nature of barriers and spin polarization of the system. In the present study with the increased $\mathrm{La}^{3+}$ doping the spin polarization should be decreasing due to unusual filling of bands as it is the common features of electron doping. Due to the lower spin polarization in electron doped system the MR values should be reduced with doping. However, the observed results are opposite and clearly state that the barriers properties (properties of grain boundaries) are playing dominant role for the observed enhancement in magnetoresistive properties.

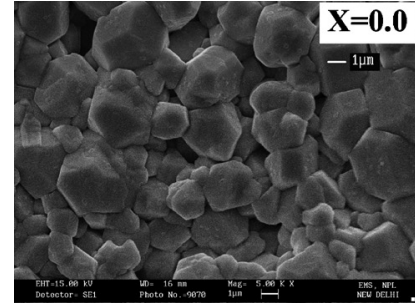

(a)

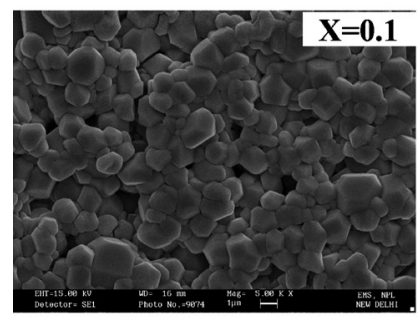

(b)

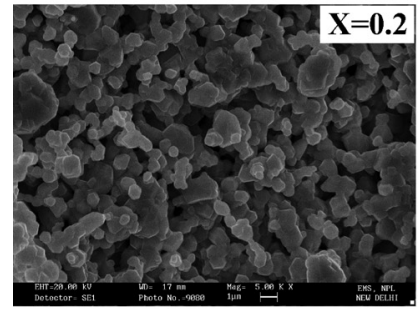

(c)

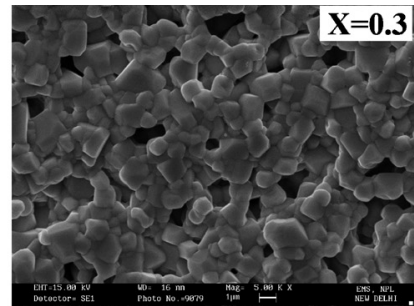

(d)

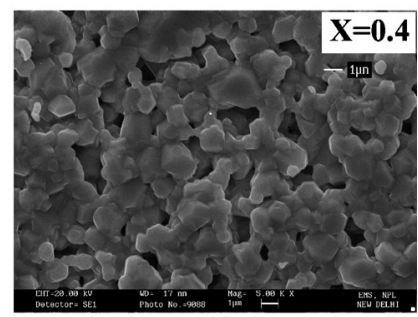

(e)

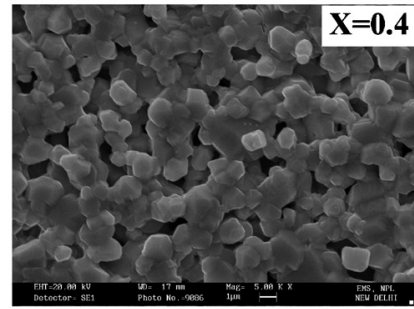

(f)
FIG. 4. [(a)-(f)] SEM images for the samples $\mathrm{Sr}_{0.4} \mathrm{Ba}_{1.6-\mathrm{x}} \mathrm{La}_{\mathrm{x}} \mathrm{FeMoO}_{6}(0.0$ $\leq \mathrm{x} \leq 0.4)$. The SEM image of sample $\mathrm{x}=0.4$ at two different places is shown.

Thus, the study of grains and grain boundaries is important at this stage. The scanning electron microscope (SEM) images for different samples are shown in Figs. 4(a)-4(f). The images revealed a heterogeneous grain growth in pristine sample with larger grains having average size more than $2.0 \mu \mathrm{m}$ and smaller grains having average grain size larger than $1 \mu \mathrm{m}$. The grain size decreases with doping thus increasing the number of grain boundary barriers. Besides the increased number of grain boundary barriers the connectivity of grains has also increased in higher doped system. Specially, the end member of series $(x=0.3$ and 0.4$)$ have more diffused grains compared to other samples. The enhancement of MR in electron doped system may be expected due to increased number of grain boundary barriers but this increase cannot solely be attributed to increased number of grain boundary barriers. The SEM images showed that the $\mathrm{x}=0.2$ and 0.3 samples has lowest average grain size of all the samples while the low temperature MR is increasing throughout the doping range. Thus the grain size alone cannot explain the observed magneto transport behavior. Further, in the SEM images it is important to note that the grain boundary connectivity is increasing with $\mathrm{La}^{3+}$ doping following the trend of increasing MR. These observations show that the connectivity of grains and the tunneling MR are well correlated with each other.

It is well known that the grain surface is a place where the off stoichiometry, vacancies, and disorders are more probable to occur. These defects acts as pinning centers for 


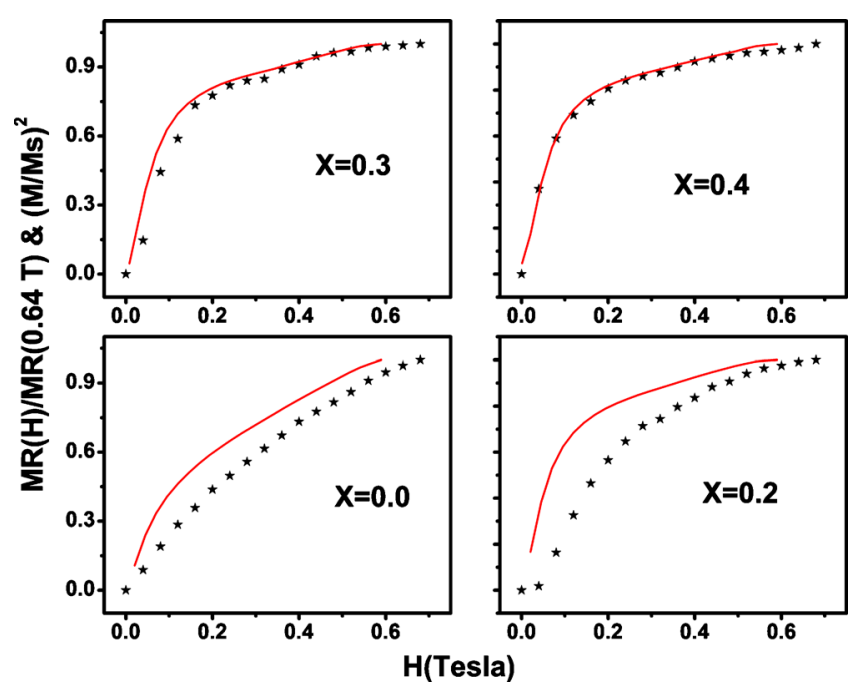

FIG. 5. (Color online) MR (H)/MR (0.64 T) vs applied field and (M/Ms) ${ }^{2}$ vs applied field plots for samples. Both MR (H)/MR $(0.64 \mathrm{~T})$ and $(\mathrm{M} / \mathrm{Ms})^{2}$ are shown at same $\mathrm{Y}$ axis.

spins giving a spin glass like behavior of grain boundaries in magnetic materials. ${ }^{14}$ The alignment of such grain boundary spins is more difficult compared to spins within the grains. Further, these defect states produces significant inelastic tunneling conductance mediated by multistep tunneling or scattering via localized defect states within barriers. ${ }^{15}$ These factors reduce the LFMR in such magnetoresistive polycrystalline systems. The probability of presence of defects states will be less in well connected grains compared to weakly connected grains. Also in the case of better connected grains the spins near grains boundaries are loosely pinned due to interactions with field of neighboring grains. The alignment of such spins is easy compared to spins in weakly connected grains. To exhibit high MR values at low fields, the spins in grain and more importantly spins at grain boundary should align easily with lower applied fields. According to conduction channel model in manganites, ${ }^{16}$ the pinned spin at grain boundaries although do not contribute for magnetization of system but blocks the conduction channel and finally reduces the LFMR of the polycrystalline system. The $\operatorname{MR}(|\Delta \rho / \rho|)$ in such grain boundary pinned spin systems fits well with $|\Delta \rho / \rho| \propto\left(\left|\mathrm{M}_{\mathrm{gb}} / \mathrm{M}_{\mathrm{g}}\right|\right)^{2}$, where $\mathrm{M}_{\mathrm{gb}}$ is the magnetization of grain boundaries and $\mathrm{M}_{\mathrm{g}}$ is magnetization of grains. In our study the room temperature MR $(\mathrm{H}) / \mathrm{MR}$ $(0.64 \mathrm{~T})$ versus field plots and the $(|\mathrm{M} / \mathrm{Ms}|)^{2}$ versus field plots runs very close in $\mathrm{x}=0.3$ and 0.4 samples, while this is not the case for other samples (Fig. 5). This led us to conclude that the grain boundary magnetization and grain magnetization approaches to each other in the samples having strongly connected grains and that such samples exhibits higher MR values at lower magnetic fields. Thus the role of grain boundary connectivity is very important for a better LFMR. The samples $\mathrm{x}=0.3$ and 0.4 with well connected grains have shown enhanced MR values at room temperature and below 1000 Oe. The weakly pinned spins align easily with lower magnetic fields and produces higher MR at lower fields in these samples. The most of MR occurs at lower field in such samples with strongly connected grains. From prac- tical point of view the main challenge lies in enhancing the low field component of MR. Our results showed that such enhancement can be achieved by manipulating the nature of grain boundaries. The most important is to note that such strategies have enough potential to enhance the LFMR value in the systems with relatively low spin polarization also. The grain boundary connectivity is one of the most important factors for such enhancement. Such strategies are more important for the double perovskite systems as the introduction of extra barriers at grain boundaries does not seem to be producing any significant improvement at room temperature although low temperature $\mathrm{MR}$ is observed to be enhanced. $^{17,18}$ Thus, the modification of barriers through grain connectivity seems to be helpful for enhancing room temperature LFMR values.

In brief, we have observed an improved low field MR values in association with a significant Curie temperature increase in an electron doped double perovskite system. The improvement in LFMR values is attributed to modification of grain boundary barriers. The results confirm the fact that the grain boundary barriers modification has enough potential to exhibit high LFMR values even in systems with relatively lower spin polarization. The grain boundary connectivity is observed to be a dominant factor for barrier modification. Finally, our results present hope toward the applicability of electron doping in these double perovskite systems and that electron doping can be utilized for increasing Curie temperature of the system and simultaneously having higher LFMR values. This may make the material beneficial to be used for various high temperature spintronics applications.

\section{ACKNOWLEDGMENTS}

Author V. Pandey acknowledges CSIR India for their fellowships. The authors are also grateful to the Director of the National Physical Laboratory, New Delhi, for providing constant encouragement and motivation to carry out this work.

${ }^{1}$ K. I. Kobayashi, T. Kimura, H. Sawada, K. Terakura, and Y. Tokura, Phys. Rev. B 59, 11159 (1999).

${ }^{2}$ D. D. Sarma, Curr. Opin. Solid State Mater. Sci. 5, 261 (2001).

${ }^{3}$ G. N. Rao, S. Roy, C.-Y. Mou, and J. W. Chen, J. Magn. Magn. Mater. 299, 348 (2006).

${ }^{4}$ D. Rubi, C. Frontera, J. Nogues, and J. Fontcuberta, J. Phys.: Condens. Matter 16, 3173 (2004).

${ }^{5}$ D. Serrate, J. M. De Teresa, J. Blasco, M. R. Ibarra, L. Morellon, and C. Ritter, Appl. Phys. Lett. 80, 4573 (2002).

${ }^{6}$ A. K. Azad, S.-G. Eriksson, A. Khan, A. Eriksson, and M. Tseggai, J. Solid State Chem. 179, 1303 (2006).

${ }^{7}$ E. K. Hemery, G. V. M. Williams, and H. J. Trodahl, J. Magn. Magn. Mater. 310, 1958 (2007).

${ }^{8}$ D. Rubi and J. Fontcuberta, J. Phys.: Condens. Matter 18, 7991 (2006).

${ }^{9}$ J. Linden, T. Shimada, T. Motohashi, H. Yamauchi, and M. Karppinen, Solid State Commun. 129, 129 (2004).

${ }^{10}$ M. Wojcik, E. Jedryka, S. Nadolski, J. Navarro, D. Rubi, and J. Fontcuberta, Phys. Rev. B 69, 100407(R) (2004).

${ }^{11}$ V. Pandey, V. Verma, R. P. Aloysius, G. L. Bhalla, and R. K. Kotnala, Solid State Commun. 149, 869 (2009).

${ }^{12}$ E. K. Hemery, G. V. M. Williams, and H. J. Trodahl, Phys. Rev. B 74, 054423 (2006).

${ }^{13}$ H. M. Yang, H. Han, and B. W. Lee, J. Magn. Magn. Mater. 272-276, 1831 (2004). 
${ }^{14}$ D. Serrate, J. M. De Teresa, P. A. Algarabel, M. R. Ibarra, and J. Galibert, Phys. Rev. B 71, 104409 (2005).

${ }^{15}$ R. Jansen and J. S. Moodera, Phys. Rev. B 61, 9047 (2000).

${ }^{16}$ A. de Andrés, M. Garcia-Hernandez, and J. L. Martinez, Phys. Rev. B 60,
7328 (1999)

${ }^{17}$ K. Wang and Y. Sui, Physica B 344, 423 (2004).

${ }^{18}$ Y. Sui, X. Zhang, X. Wang, J. Cheng, W. Su, and J. Tang, J. Appl. Phys. 102, 023903 (2007). 